\title{
Effects of the Insulation Quality on the Frequency Response of Power Transformers
}

\author{
Nilanga Abeywickrama*, Chandima Ekanayake**, \\ Yuriy V. Serdyuk* and Stanislaw M. Gubanski*
}

\begin{abstract}
This paper presents results of frequency domain spectroscopy (FDS) measurements on oilimpregnated pressboard insulation, their analyses and use of the data for modeling high frequency response (FRA) of transformers. The dielectric responses were measured in a broad frequency range, i.e. from $0.1 \mathrm{mHz}$ to $1 \mathrm{MHz}$, on model samples containing different amount of moisture. The responses were parameterized with terms representing dc conductivity, low frequency dispersion and Cole-Cole polarization mechanisms and they were thereafter used to model the FRA response of a three-phase transformer.
\end{abstract}

Keywords: Frequency domain spectroscopy, Frequency response analysis, Insulation modeling, Moisture content, Transformer insulation

\section{Introduction}

Among factors influencing degradation of oilimpregnated pressboard/paper insulation in power transformers, moisture is of the highest significance. Therefore, an early identification of moist insulation is of vital importance for avoiding sudden failures. Different measuring techniques and interpretation schemes have been developed to evaluate the moisture content [1]. Among the techniques, the frequency domain spectroscopy (FDS) measurement is the one, which have become especially popular and attractive. However, to utilize this method effectively, a modeling based on good knowledge on the effects of moisture on the dielectric response of pressboard and paper is necessary.

Frequency Response Analysis (FRA) has been used as a tool for diagnosing mechanical faults inside transformers, based on the fact that the frequency response is dependent on the values of capacitances and inductances, which are mainly defined by geometrical parameters. However, the FRA signal carries information on the insulation condition as well. Although the physical dimensions of transformer define the geometrical capacitances, changes in the complex permittivity of the insulation system modify it also.

In this paper results of investigations on the influence of

* Division of High Voltage Eng., Dept. of Materials and Manufacturing Technology, Chalmers University of Technology, Sweden.

(nilanga.abeywickrama@chalmers.se, yuriy.serdyuk@chalmers.se, stanislaw.gubanski@chalmers.se )

** Dept. of Electrical and Electronic Engineering, Faculty of Engineering, University of Peradeniya, Peradeniya, Sri Lanaka.

(chandima@ee.pdn.ac.lk)

Received June 19, 2006 ; Accepted October 14, 2006 moisture on the dielectric response characteristics of pressboard and paper in a broad frequency range are presented. Thereafter, these data are used to illustrate how the insulation quality may influence the high frequency $(100 \mathrm{~Hz}-1 \mathrm{MHz})$ response of a distribution transformer (400 kVA, $15 / 0.4 \mathrm{kV}, \Delta-\mathrm{Y}$ ). The measurements were performed by means of a network analyzer with a reflection bridge, while the primary and the secondary terminals were tied together.

\section{Frequency Dependent Dielectric Response of Impregnated Paper/pressboard}

Laboratory measurements were carried out to obtain the frequency dependent dielectric responses of wellcontrolled impregnated samples. Pressboard/paper samples having different moisture contents (i.e. $1 \%, 2 \%, 3 \%$ and $4 \%$ ) were selected for the investigation. Some of the samples having $2 \%$ and $4 \%$ moisture content were also artificially aged. The frequency domain spectroscopy (FDS) measurements on all the selected samples were performed at three different temperatures, i.e. $20^{\circ} \mathrm{C}, 50^{\circ} \mathrm{C}$ and $80^{\circ} \mathrm{C}$. Detailed information on the sample preparation and on the performed measurements can be found in [2]

\subsection{Dielectric Response of Unaged Pressboard}

Average dielectric responses at $50{ }^{\circ} \mathrm{C}$ of pressboard samples having similar moisture contents are illustrated in Fig. 1. Similar characteristics in the frequency range of $1 \mathrm{mHz}-1 \mathrm{MHz}$, though shifted in the frequency scale, could also be seen at $20^{\circ} \mathrm{C}$ and $80^{\circ} \mathrm{C}$. 

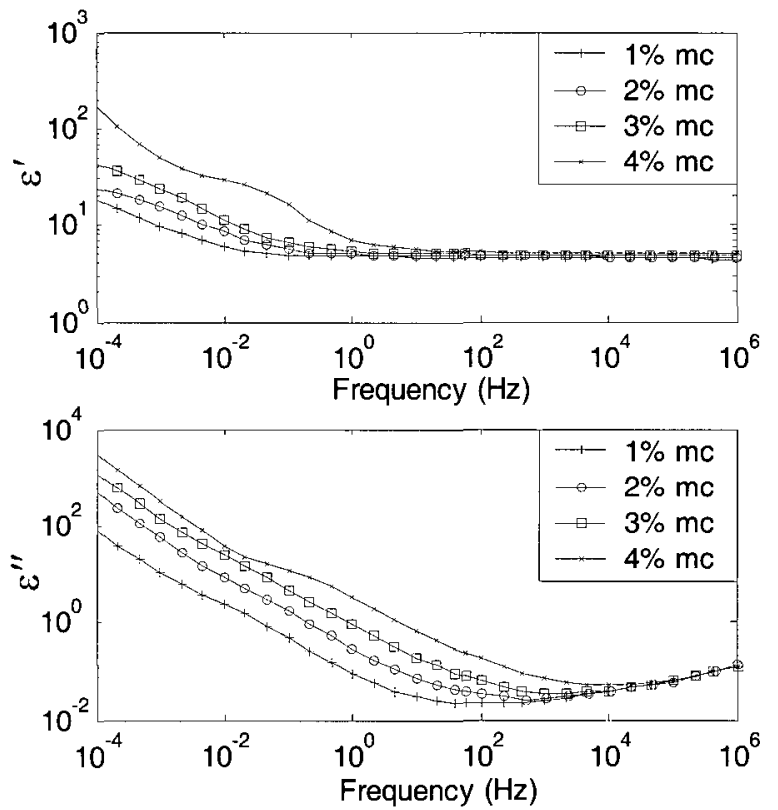

Fig. 1 Average dielectric responses at $50^{\circ} \mathrm{C}$ of pressboard containing different moisture contents.

The dielectric losses $\left(\varepsilon^{\prime \prime}\right)$ increase significantly below $100 \mathrm{~Hz}$ with the increasing moisture content. At the same time, there is no much influence of the humidity on the loss characteristics at frequencies higher than $10 \mathrm{kHz}$. In general, the losses increase above this frequency limit is very weakly dependent on moisture content. It also looks as if another loss peak is present at much higher frequencies. The permittivity $\left(\varepsilon^{\prime}\right)$ also increases with decreasing frequency below $1 \mathrm{~Hz}$ and this behavior appears to be sensitive to moisture content. At frequencies above $100 \mathrm{~Hz}$, a constant and weak increase in permittivity can be observed with increasing moisture content, though it is not as significant as in the low frequency range.

Fig. 2 shows the dielectric responses of impregnated pressboard having $2 \%$ moisture content. Since the spectral shape of the responses does not change with temperature, a master curve can be formed by shifting the spectra along the frequency axis until they coincide into a single curve. The shifts of the spectra were used to calculate the activation energies, by assuming the processes follow Arrhenius type of temperature dependence. The calculated activation energies are presented in Table I. The master curve for pressboard with $2 \%$ moisture intake at $20{ }^{\circ} \mathrm{C}$ is also illustrated in Fig. 2. The obtained master curve provides information about the involved polarization processes in a broader frequency range ( 13 decades).

The behavior of low frequency part of permittivity $\varepsilon^{\prime}$ was modeled by an inverse power dependence on frequency, $A \omega^{n}$, whereas the remaining part was modeled by Cole-Cole expressions with two distinct relaxation times $\tau_{1}$ and $\tau_{2}$ [3]. The total relaxation can therefore be represented as indicated in equation (1).
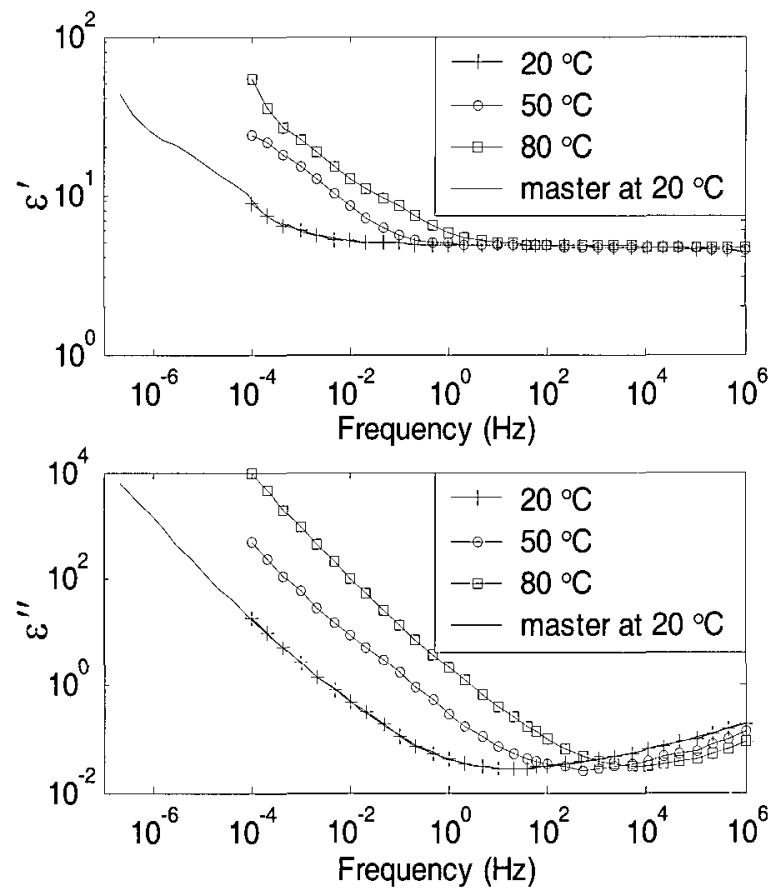

Fig. 2 Average dielectric responses at different temperatures for pressboard containing $2 \%$ moisture intake and the corresponding master curves.

The imaginary part of the complex permittivity $\varepsilon^{\prime \prime}{ }_{\text {tot }}$, denoted in equation (2), was calculated by the addition of dc losses to Kramers-Kronig (K-K) transformation of (1) [3].

$$
\begin{aligned}
\varepsilon^{\prime}= & A \omega^{-n}+\operatorname{Re}\left\{\frac{\Delta \varepsilon_{1}}{1+\left(\mathrm{j} \omega \tau_{1}\right)^{1-\alpha_{1}}}+\frac{\Delta \varepsilon_{2}}{1+\left(\mathrm{j} \omega \tau_{2}\right)^{1-\alpha_{2}}}\right\}+\varepsilon_{\infty} \\
\varepsilon_{t o t}^{\prime \prime} & =\frac{\sigma_{d c}}{\varepsilon_{0} \omega}+A \omega^{-n} \cot \left((1-n) \frac{\pi}{2}\right)+ \\
& +\operatorname{Im}\left\{\frac{\Delta \varepsilon_{1}}{1+\left(\mathrm{j} \omega \tau_{1}\right)^{1-\alpha_{1}}}+\frac{\Delta \varepsilon_{2}}{1+\left(\mathrm{j} \omega \tau_{2}\right)^{1-\alpha_{2}}}\right\}
\end{aligned}
$$

The parameters $A, n, \alpha_{1}, \alpha_{2}, \tau_{1}, \tau_{2}, \Delta \varepsilon_{1}, \Delta \varepsilon_{2}$ and $\sigma_{d c}$ in (1) and (2) were estimated by means of the least square technique to obtain the best fit of the measured permittivity and losses. As we do not have any information about the response of pressboard at very high frequencies, $\varepsilon_{\infty}$ was assumed to be equal to 1 . Fig. 3 represents the modeled curves of complex permittivity together with the corresponding measured results on impregnated pressboard with $4 \%$ moisture content at $50{ }^{\circ} \mathrm{C}$. It is clearly seen that the modeled curves are well-matching the measured responses. A similar fit between the modeled and the measured responses was observed at the three different temperature levels. 

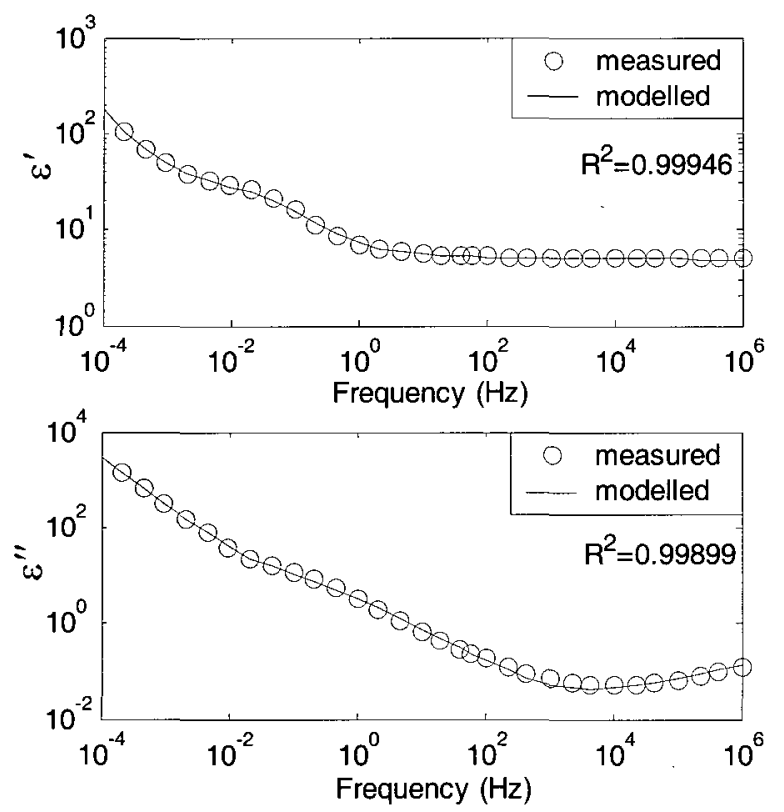

Fig. 3 Measured and modeled (eqns. (1) and (2)) curves of permittivity and loss at $50^{\circ} \mathrm{C}$ of pressboard sample with $4 \%$ moisture intake.

Numerical values of the estimated model parameters for the dielectric responses at $50{ }^{\circ} \mathrm{C}$ of the pressboard having different moisture content are listed in Table I. In the table column named "Comments" the observed variations of each parameter with respect to changing temperature and moisture content are described. Parameter A, which represents intensity of the low frequency dispersion process, and the dc conductivity $\sigma_{d c}$, both become more pronounced with increasing the temperature and the moisture content. At the same time, the parameters of the first Cole-Cole process, especially $\tau_{I}$ are also highly sensitive to the changes of temperature and moisture content. $\tau_{1}$ significantly decreases with the rise in temperature. The time constant of the second Cole-Cole process $\tau_{2}$, also decreases with increasing temperature. On the other hand, increasing moisture content causes a slight increment of $\tau_{2}$.

\subsection{Dielectric Response of Aged Pressboard}

The average dielectric responses of aged and new pressboard samples having two different moisture intakes (i.e. $2 \%$ and $4 \%$ ) are presented in Fig. 4 . In this figure, one can clearly see that the process of ageing yields an increment and change in the spectral shape of both the real
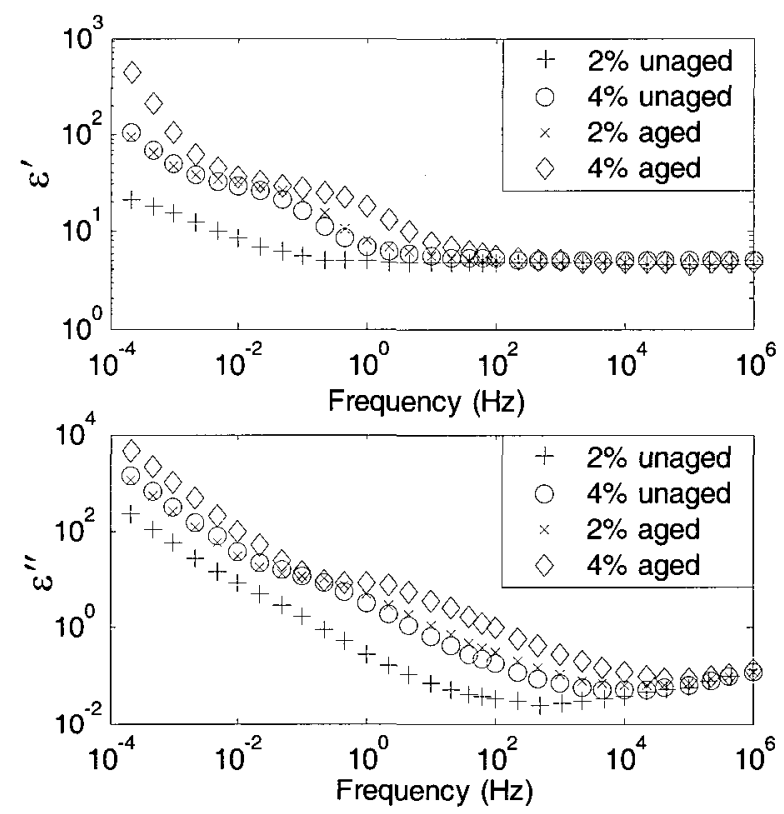

Fig. 4 Frequency response of aged and unaged pressboard samples at $50^{\circ} \mathrm{C}$.

Table 1 Estimated model parameters for responses at $20^{\circ} \mathrm{C}$ of the pressboard samples having different moisture contents

\begin{tabular}{|c|c|c|c|c|c|}
\hline \multirow{2}{*}{ Parameter } & \multicolumn{4}{|c|}{ Moisture content $(\%)$} & \multirow{2}{*}{ Comments } \\
\hline & 1 & 2 & 3 & 4 & \\
\hline$A$ & 0.073 & 0.076 & 0.200 & 0.210 & Increases with increasing temperature and moisture content \\
\hline$n$ & 0.71 & 0.6 & 0.61 & 0.89 & $\begin{array}{l}\text { Increases with temperature, typical value around } 0.6 \text { and } \\
\text { always less than } 1\end{array}$ \\
\hline$\Delta \varepsilon_{1}$ & 2.2 & 15.0 & 23.0 & 25.0 & Increases with increasing moisture content \\
\hline$\tau_{l}(\mathrm{~s})$ & 35 & 100 & 72 & 25 & Decreases with increasing temperature \\
\hline$\alpha_{1}$ & 0.34 & 0.39 & 0.44 & 0.33 & $\begin{array}{l}\text { No clear variation with changing temperature and moisture } \\
\text { content }\end{array}$ \\
\hline$\Delta \varepsilon_{2}$ & 3.7 & 3.8 & 3.9 & 4.1 & $\begin{array}{l}\text { Almost no variation with temperature, slight increase with } \\
\text { moisture content }\end{array}$ \\
\hline$\tau_{2}(\mathrm{~s}) \times 10^{-12}$ & 8 & 10 & 30 & 31 & $\begin{array}{l}\text { Decreases with increasing temperature, tendency to increase } \\
\text { with increasing moisture content }\end{array}$ \\
\hline$\alpha_{2}$ & 0.76 & 0.75 & 0.71 & 0.71 & $\begin{array}{l}\text { Slightly decreases with increasing temperature and moisture } \\
\text { content }\end{array}$ \\
\hline$\sigma_{d c}(\mathrm{~S} / \mathrm{m}) \times 10^{-12}$ & 0.29 & 2.73 & 7.11 & 12.02 & Increases with temperature and moisture content. \\
\hline $\begin{array}{l}\text { Activation energy } \\
(\mathrm{eV})\end{array}$ & 0.70 & 0.72 & 1.00 & 0.90 & Tendency to increase with increasing moisture content \\
\hline
\end{tabular}


and the imaginary parts of the complex permittivity when the frequency is between $0.1 \mathrm{mHz}$ and $100 \mathrm{~Hz}$. However, it is not clear yet, if these changes are caused by reducing depolymerisation degree of the pressboard or by appearance of ageing by-products, such as acids or water.

The responses of aged samples were also used to estimate the dc conductivity by means of fitting eqns. (1) and (2). According to these estimates the dc conductivity of pressboard has increased with ageing by a factor varying between 1.4 and 6 . One possible reason for this increase could be the influence of acids formed in the pressboard.

\subsection{Dielectric Response of Paper}

Fig. 5 presents the measured frequency responses at 50 ${ }^{\circ} \mathrm{C}$ of both pressboard and paper having two different moisture intakes $(1 \%$ and $3 \%)$. As seen in the figure, the permittivity of paper is slightly lower than that of pressboard throughout the measured frequency span. This is mainly due to the higher density of pressboard as it is formed by wet pressing of paper. Furthermore, at some frequencies the loss in paper is slightly lower than that of pressboard. However, the responses of both materials exhibit similar spectral shapes. The expressions (1) and (2) were therefore used to model the responses of paper samples. A good agreement between the measured and modeled results was obtained. The dc conductivity of paper was also estimated and the results revealed that the conductivity values for paper and pressboard were in most of the cases within the same range, though pressboard was always having a higher value.
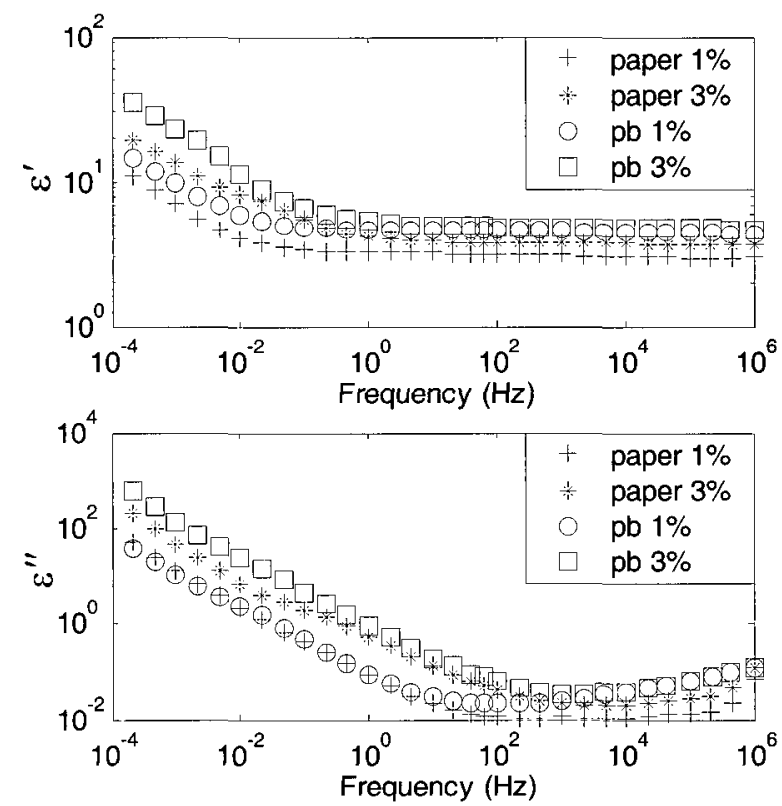

Fig. 5 Comparison of the measured permittivity and loss of paper and pressboard (pb) at $50{ }^{\circ} \mathrm{C}$.
Furthermore, for samples having $2 \%$ and $3 \%$ moisture content, the ratio of the conductivities of pressboard and paper was at different temperatures almost identical $(\sim 0.3)$.

The measured dielectric response data were incorporated into the analyses presented in the next section for identifying the influence of the pressboard conditions on FRA results of power transformers.

\section{Modeling 3-phase Transformers for High Frequency Range}

For modeling the high frequency response of power transformer, windings can be split into finite sections, each of them being represented by its own lumped capacitance, conductance, inductance and resistance. The size of the section should be small enough to assume that the current flowing through it is constant [4] and the lower limit of the size of a section is usually determined by the desired bandwidth of the model and the geometry of the windings. In the particular case studied here, each disk of the HV disk winding and a single turn of the LV helical winding were treated as a section.

In Fig. 6 a lumped circuit consisting of passive elements ( $C, R, L$ and $G$ ) representing a single phase transformer is shown with both windings divided into ' $n$ ' sections.

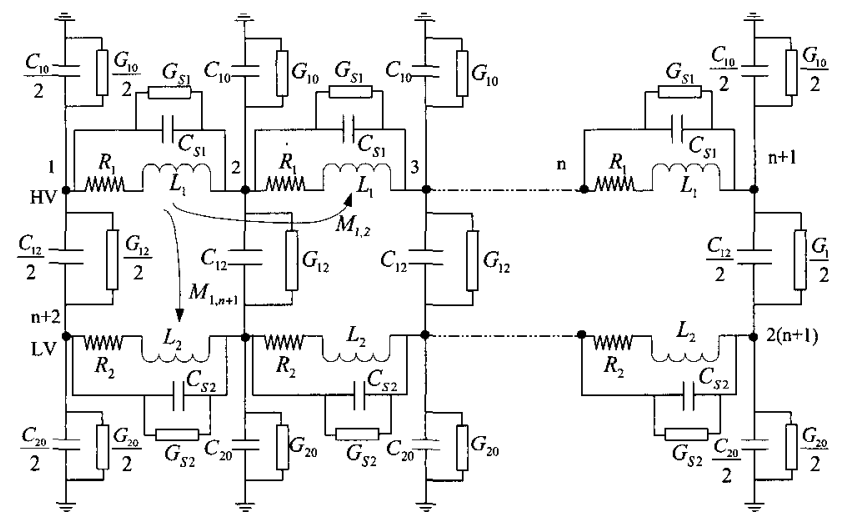

Fig. 6 parameter circuit model of a two winding transformer.

The circuit elements marked are:

$\mathrm{C}_{10}, \mathrm{C}_{20}$ - capacitances of the windings to earth,

$\mathrm{G}_{10}, \mathrm{G}_{20}$ - conductances between earth and the windings,

$\mathrm{C}_{12}, \mathrm{G}_{12}$ - capacitance and conductance between windings,

$\mathrm{C}_{\mathrm{S} 1}, \mathrm{C}_{\mathrm{S} 2}$ - series capacitances of the windings,

$\mathrm{G}_{\mathrm{S} 1}, \mathrm{G}_{\mathrm{S} 2}$ - series conductances of the windings,

$L_{1}, R_{1}$ and $L_{2}, R_{2}$ - inductances and equivalent resistances of the windings,

$\mathrm{M}_{\mathrm{i}, \mathrm{j}}$ - mutual inductance between sections $\mathrm{i}$ and $\mathrm{j}$. 
Dynamics of the lumped circuit can be mathematically modeled using state-space representation of a Linear Time Invariant (LTI) system [5], in which the state vector consists of all the nodal voltages and the inductor currents. There are $2(n+1)$ nodes in the circuit, $2 n$ inductive branches and associated capacitances and conductances. By considering the voltage difference between two nodes of each inductive branch, one can write

$$
\begin{aligned}
{[\Gamma]_{2(n+1) \times 2 n}[I]_{2 n \times 1}=} & {[C]_{2(n+1) \times 2(n+1)}[\dot{V}]_{2(n+1) \times 1} } \\
& +[G]_{2(n+1) \times 2(n+1)}[V]_{2(n+1) \times 1}
\end{aligned}
$$

Conservation of currents at each node is

$$
-\left[\Gamma^{T}\right]_{2 n \times 2(n+1)}[V]_{2(n+1) \times 1}=[L]_{2 n \times 2 n}[\dot{I}]_{2 n \times 1}+[R]_{2 n \times 2 n}[I]_{2 n \times 1} .
$$

In equations (3) and (4), $V$ and $\dot{V}$ are column matrices that represent nodal voltages and their time derivatives respectively, currents through the inductors and their time derivatives are represented by matrices $I$ and $\dot{I} . C$ and $G$ include capacitances and conductance respectively, and $L$ and $R$ contain inductances (self and mutual) and resistances respectively. The matrix $\Gamma$ (consisting of 1's, 1 's and 0 's) connects the current and voltage equations. When an external voltage source is connected to a node (node $k$ ), it is introduced into the equations (3) and (4) as an input voltage accompanied by corresponding columns of matrices $C$ and $G(Q)$. In addition, corresponding rows with index ' $k$ ' in matrices $\Gamma, C$ and $G$ should be removed.

$$
\begin{aligned}
{[\Gamma]_{(2 n+1) \times 2 n}[I]_{2 n \times 1}=} & {[C]_{(2 n+1) \times(2 n+1)}[\dot{V}]_{(2 n+1) \times 1} } \\
+ & {[G]_{(2 n+1) \times(2 n+1)}[V]_{(2 n+1) \times 1}+[Q]_{(2 n+1) \times 2}\left[V_{k}\right]_{(2 \times 1)} } \\
{[P]_{(2 n+1) \times 2}\left[V_{k}\right]_{2 \times 1}-\left[\Gamma^{T}\right]_{2 n \times(2 n+1)}[V]_{(2 n+1) \times 1}=} & {[L]_{2 n \times 2 n}[\dot{I}]_{2 n \times 1} } \\
& +[R]_{2 n \times 2 n}[I]_{2 n \times 1}
\end{aligned}
$$

Apart from aforementioned changes, further alterations to equations (5) and (6) must be made, depending if winding ends are grounded or connected together (in three phase configuration). These alterations are listed below.

1. Connecting two nodes together: adding up the corresponding rows and columns in $C$ and $G$ matrices respectively and adding only corresponding rows of matrix $\Gamma$.

2. Grounding a node: removing corresponding rows and columns in $C$ and $G$ matrices and removing only the corresponding column in matrix $\Gamma$.
By rearranging the terms in (5) and (6), one obtains

$$
\begin{aligned}
& \dot{V}=-C^{-1} G V+C^{-1} \Gamma I-C^{-1} Q V_{k} \\
& \dot{I}=-L^{-1} \Gamma^{T} V-L^{-1} R I+L^{-1} P V_{k}
\end{aligned}
$$

These two equations can be transformed into Multi Input Multi Output (MIMO) state space model of the lumped parameter circuit as follows:

$$
\dot{X}=A X+B V_{k}
$$

where $X=\left[\begin{array}{l}V \\ I\end{array}\right], \quad A=\left[\begin{array}{cc}-C^{-1} G & C^{-1} \Gamma \\ -L^{-1} \Gamma^{T} & -L^{-1} R\end{array}\right]$ and $B=\left[\begin{array}{c}-C^{-1} Q \\ L^{-1} P\end{array}\right]$.

Laplace transform of (9) and selection of all state variables as outputs yields

$$
H(s)=\frac{X(s)}{V_{k}(s)}=[s I I-A]^{-1} B .
$$

Where, $s$ is Laplace operator and $I I$ is the identity matrix the latter has number of columns and rows equal to the size of the state matrix $X$. Equation (10) represents the transfer functions of all the nodal voltages and the inductor currents with reference to the applied voltage $\left(V_{k}\right)$.

A three-phase transformer can be considered as three sets of single-phase units (Fig. 6) connected into either delta or star configuration. For example, Fig. 7 shows three single-phase units connected in $\mathrm{Y}-\mathrm{Y}$ configuration.

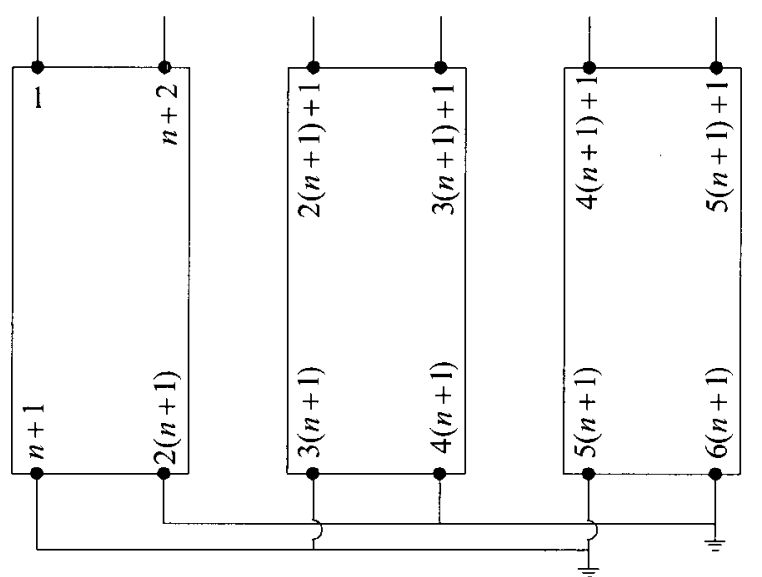

Fig. 7 Three phase circuit model connected in $Y-Y$ configuration.

In case of a three phase transformer, the matrices $L, R, C$ $G$ and $\Gamma$ should be modified in order to account for the mutual coupling among the windings on different limbs of transformer [6]. For a three-phase transformer, number of columns and rows in aforementioned matrices become 
three times lager than that of a single-phase case. The developed state space model for the three phase transformer was then implemented in $\mathrm{Matlab}^{(8)}$ and analyzed for different frequencies $(s=j \omega)$.

Elements in matrices $L, R, C$ and $G$ include all the frequency dependent parameters related to the windings, the core and the insulation. Calculations of the circuit parameters of the lumped circuit model and implementation of the model are elaborated in [6]. The model was used to simulate the frequency response of the impedance between the primary and the secondary windings of the studied transformer in the range of $100 \mathrm{~Hz}$ $-1 \mathrm{MHz}$, which hereafter is called as transformer response. It was analyzed to investigate the influence of insulation quality (temperature, moisture content, etc).

The simulated and the measured responses are compared in Fig. 8. Initial slope of the transformer response $(-1$ in $\log -\log$ scale) appears due to the capacitance of the main insulation $\left(\mathrm{C}_{12}\right)$ of the transformer, which consists of pressboard barriers and oil ducts. $C_{12}$ can therefore be represented by the $X Y$-model [7]. The first deep valley is due to a series resonance between the capacitance of the main insulation and the effective inductance of the windings created by tying HV and LV terminals together. Then the main peak and the following series of minor peaks are resulting from the serial capacitances and inductances along $\mathrm{HV}$ winding.

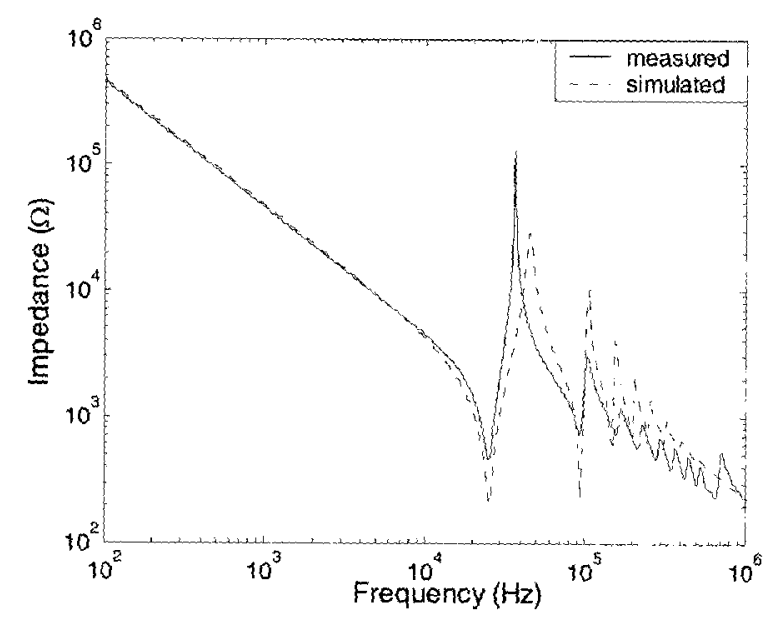

Fig. 8 Measured and simulated responses of the impedance between primary and secondary windings.

The observed discrepancies between the measured and the simulated responses could be explained as inaccuracies when calculating capacitances, inductances and losses. The capacitances among the windings on different limbs, which are not included in the model, could also affect the obtained result. The secondary slope with superimposed minor peaks above $100 \mathrm{kHz}$ is defined by the serial capacitances of the HV winding. It becomes therefore clear that the estimated $C_{\mathrm{S} 1}$ value could be lower than the real one.

\section{Influence of Insulation Quality on High Frequency Response}

The measured dependences of complex relative permittivity of paper and pressboard were used in this analysis. Since the differences in complex permittivity appearing at different conditions (moisture content, temperature and aging) at higher frequencies are not seen clearly in Figs. 1,2 and 4, an exaggerated view of these influences in the frequency range $100 \mathrm{~Hz}-1 \mathrm{MHz}$ is presented in Figs. 9 and 10. One can clearly realize from the figures that moisture mostly affects the real part of permittivity whereas aging and temperature variations influence mostly the imaginary part.

\subsection{Influence of Temperature}

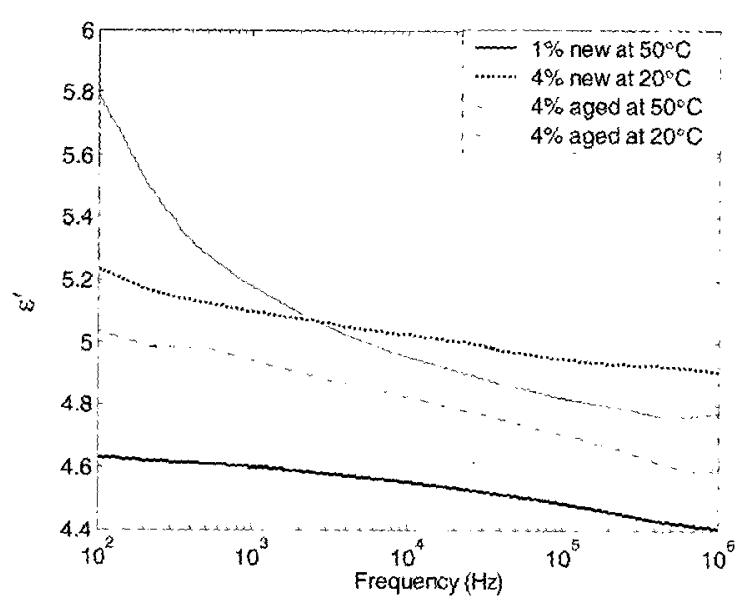

Fig. 9 Real part of the complex permittivity of pressboard in different conditions.

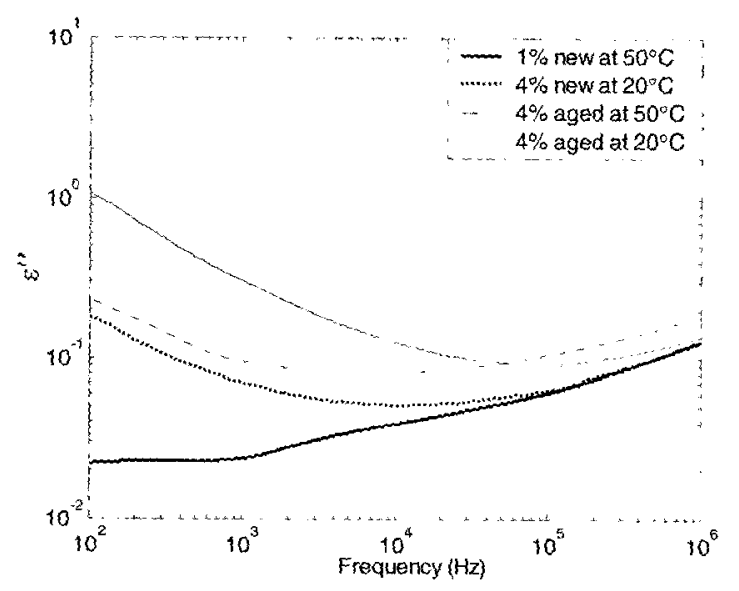

Fig. 10 Imaginary part of the complex permittivity of pressboard in different conditions. 
Since the influence of temperature on the real part of complex permittivity is rather small and the imaginary part (losses) varies with the temperature much stronger (compare Figs. 9 and 10), the influence of temperature on the transformer response can mainly be seen as a change in damping of the resonances. It is also observed that the damping increases when the moisture content is high, since the losses in the insulation are higher. Fig. 11 depicts the simulated results for three different temperature values at $4 \%$ moisture content.

\subsection{Influence of Aging}

Aging also does not influence significantly the real part of complex permittivity, which results in barely visible change in the transformer response. However it influences the losses, yielding higher damping of resonant frequencies, similarly as in the case of temperature dependence. Fig. 12 depicts the influence of aging of paper and pressboard insulation with $4 \%$ moisture content at $80^{\circ} \mathrm{C}$.

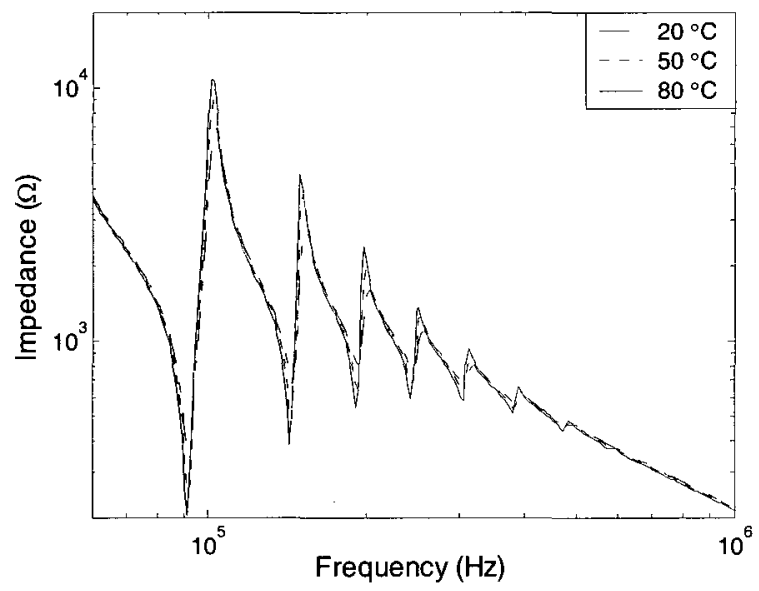

Fig. 11 Influence of temperature on the frequency response.

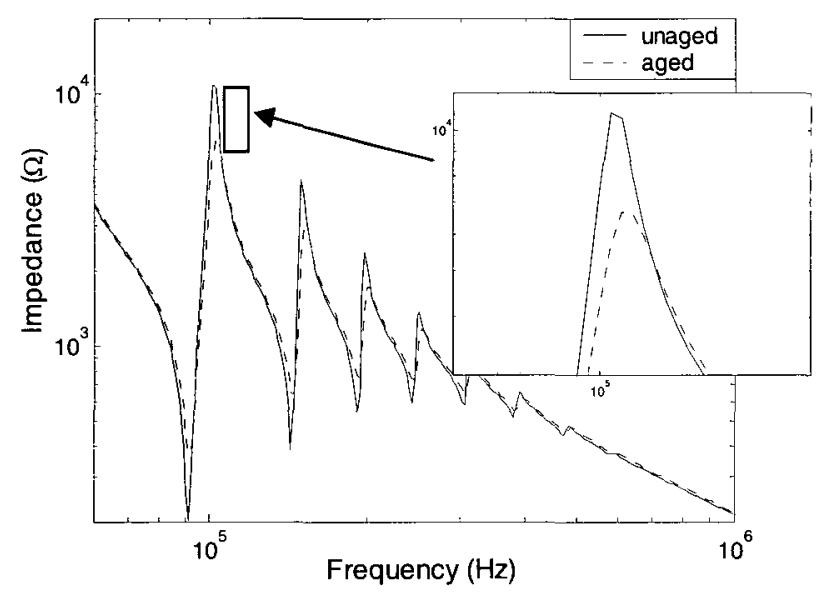

Fig. 12 Influence of aging of the insulation on the frequency response.

\subsection{Influence of Moisture Content}

Moisture content is the most influencing parameter, which changes both real and imaginary parts of complex permittivity. Therefore, changes in moisture content can distinctively be seen as shifts of the resonant peaks or the valleys in the transformer response. As shown in Fig. 13, the whole transformer response shifts to the left along the frequency scale, when the moisture content rises from $1 \%$ to $4 \%$. The higher the moisture content the higher the real part of the complex permittivity (see Fig. 9), which increases the value of capacitances and results in the lower resonant frequencies. For example, the first peak is shifted by about $1 \mathrm{kHz}$. The frequency shift is not constant over the whole frequency range. The absolute frequency shift gets higher with the increasing frequency while the percentage shift remains more or less similar [6]. Furthermore, it must be mentioned that all the capacitances in the lumped circuit model are not affected in the same proportion by the change in the real part of complex permittivity. For example, the change in $\mathrm{C}_{12}$, consisting of oil, paper and pressboard, is qualitatively not the same as the change in $\mathrm{C}_{\mathrm{S} 1}$, which consists mainly of paper and oil.

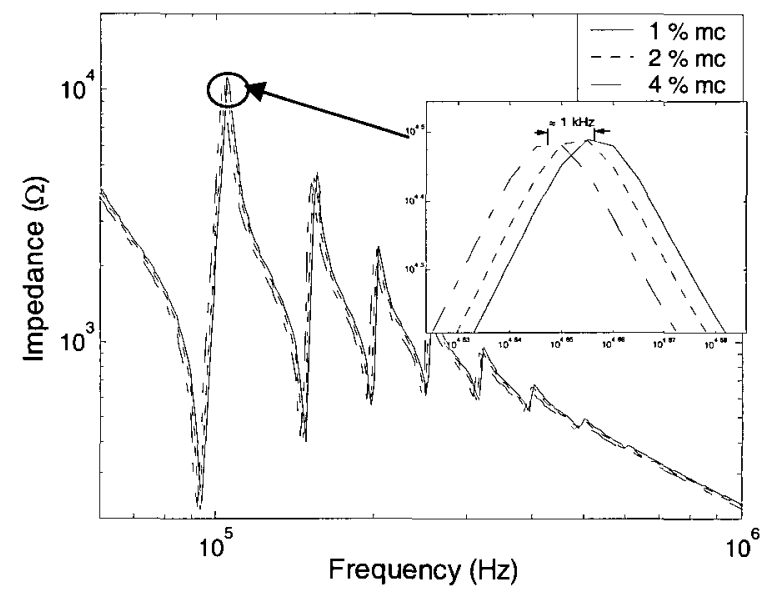

Fig. 13 Influence of moisture content of the insulation on the frequency response.

\section{Conclusion}

Influence of moisture in impregnated paper/pressboard insulation is much stronger manifested at very low frequencies $(<1 \mathrm{~Hz})$ than at higher frequency range $(>100$ $\mathrm{Hz}$ ). In the later case a constant increase of permittivity $\varepsilon^{\prime}$ is found with the increase of moisture content. At the same time, the influence of moisture on the loss $\varepsilon^{\prime \prime}$ is more pronounced and visible in the whole frequency range below $1 \mathrm{kHz}$. Similar responses are observed for both 
impregnated paper and pressboard, although there exist small differences, mainly caused by differences in material density.

All the measured dielectric responses can be modeled by a combination of formulas representing frequency dependences of de conductivity, low frequency dispersion and Cole-Cole polarization mechanisms. The Cole-Cole polarizations consist of two distinct relaxation processes with two time constants, of which the longer one decreases with both, the increasing temperature and the increasing moisture content. On the other hand, the shorter time constant decreases with the increasing temperature; it also decreases slightly with the increasing moisture content.

As regards the modeling the high frequency response of transformers, the change in moisture content is mostly manifested as a shift in resonant frequencies. However the other two factors influencing the dielectric responses of impregnated paper and pressboard, i.e. temperature and aging, show only up as damping of the resonances, mostly because they are influenced by the changes of the dielectric loss. One may therefore state that very precise measurements would have to be performed for detecting small deviations in the transformer high frequency response for tracing the changes of insulation quality. Such accuracy is however unavailable today.

\section{Acknowledgements}

This work was supported by the European Union Project RADIATOOL ("Reliable Diagnostics of HV transformer Insulation for Safety Assurance of Power Transmission Systems"- Contract no. NNE5/2001/472). Sida/SAREC of Sweden has also contributed via financing a $\mathrm{PhD}$ student at Chalmers University of Technology.

\section{References}

[1] S. M. Gubanski, P. Boss, G. Csepes, V. Der Houhanessian, J. Filippini, P. Guuinic, U. Gäfvert, V. Karius, J. Lapworth, G. Urbani, P. Werelius and W. Zaengl, "Dielectric response methods for diagnostics of power transformers", IEEE Electrical Insulation Magazine, Vol. 19, No. 3, pp. 12-18, 2003. (also in CIGRE Technical brochure No. 254, Paris 2004).

[2] C. Ekanayake, "Diagnosis of moisture in transformer insulation - Application of frequency domain spectroscopy", Ph.D. thesis, ISBN 91-7291-727-X, Chalmers University of technology, Gothenburg, Sweden 2006.
[3] A. K. Jonscher, Dielectric relaxation in solids, 2nd ed. London, UK, Chelsea Dielectrics Press Limited, 1996.

[4] P. Holmberg, "Modeling the transient response of windings, laminated steel cores and electromagnetic power devices by means of lumped circuits", $\mathrm{PhD}$ Thesis, ISBN 91-554-4877-1, Uppsala University, Uppsala, Sweden, 2000.

[5] N. S. Nise, Control System Engineering. New York: John Wiley \& Sons, Inc, 2000.

[6] Nilanga Abeywickrama, "Modeling of high frequency response of transformers for characterization of insulation quality", Licentiate thesis, ISSN 1652-8891, Chalmers University of technology, Gothenburg, Sweden 2005.

[7] U. Gafvert, G. Frimpong, and J. Fuhr, "Modeling of dielectric measurements of power transformers", paper 15-103, 1998 CIGRE session, Paris, 1998.

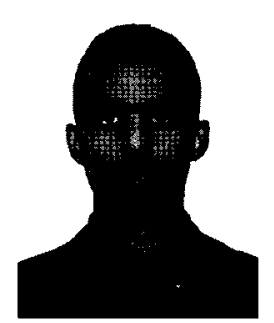

\section{Nilanga Abeywickrama}

He received the B.Sc. degree in electrical and electronic engineering from the University of Peradeniya, Sri Lanka, in 2001, M.Sc. degree in electrical power and energy from the same university, in 2003, and the Lic. Eng. degree in 2005 from the Division of High Voltage Engineering, Chalmers University of Technology (CTH), Göteborg, Sweden, where he is currently pursuing the Ph.D. degree . His research interests include high frequency modeling, high frequency measurements and diagnosis of power transformers.

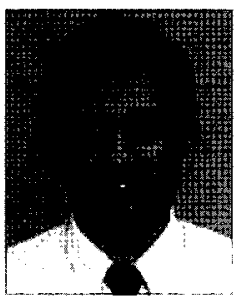

\section{Chandima Ekanayake}

$\mathrm{He}$ received the B.Sc. degree in electrical and electronic engineering from University of Peradeniya, Sri Lanka, in 1999. He obtained the Tech. Lic. degree and Ph.D. in HV Engineering from Chalmers University of Technology (CTH), Gothenburg, Sweden, in 2003 and 2006 respectively. Currently he is a senior lecturer at the Department of Electrical and Electronic Engineering in University of Peradeniya, Sri lanka. His current research work is on diagnostics of power transformer insulation by dielectric response. 


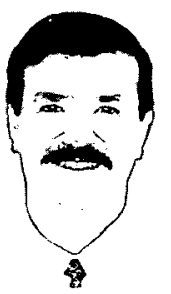

\section{Yuriy V. Serdyuk}

He received the M.Sc. (HV Engineering) and Ph.D. (HV Engineering) degrees from the National Technical University of Ukraine "Kiev Polytechnic Institute" Kiev, Ukraine in 1986 and 1995, respectively. From 1986, he worked as an engineer, and since 1991, as a researcher at the institute of Electrodynamics of the National Academy of Science of Ukraine Kiev, Ukraine. In 1996-1997 he was a researchengineer at the R \& D HV Laboratory, ABB High Voltage Technologies Ltd, Zurich, Switzerland. Currently, he is an assistance professor at the Division of High Voltage Engineering, Department of Manufacturing and Materials Technology, Chalmers University of Technology, Göteborg, Sweden.

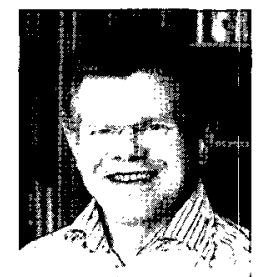

\section{Stanislaw M. Gubanski}

$\mathrm{He}$ received the M.Sc. (high voltage engineering) and Ph.D. degrees (material science) from the Technical University of Wroclaw, Wroclaw, Poland, in 1973 and 1976, respectively. He was a Research Fellow at the University College of North Wales Bangor, U.K from 1967 to 1977 , a senior lecturer at the technical university of Wroclaw, Wroclaw, Poland, from 1977 to 1988 . He was an associate professor in the Department of Electric Power Engineering, Royal Institute of Technology, Stockholm, Sweden. Currently, he is a Professor in the Division of High Voltage Engineering, Department of Manufacturing and Materials Technology, Chalmers University of Technology, Göteborg and research leader of High Voltage Valley in Ludvika, Sweden. He is IEEE Fellow and Chair of IEEE-DEIS Nominations Committee. He is also convener of the CIGRE Task Force D1.01.14 "Dielectric Response Methods for Diagnostics of Power Transformers". 